\title{
Composición de especies y diversidad de peces en un cuerpo de agua temporal en el Refugio Nacional de Vida Silvestre Caño Negro, Costa Rica
}

\author{
Idania Sáenz Sánchez, Maurizio Protti Quesada \& Jorge Cabrera Peña \\ Laboratorio de Recursos Naturales y Vida Silvestre, Escuela de Ciencias Biológicas, Universidad Nacional, Heredia \\ 86-3000, Costa Rica. Idania11@Latinmail.com o mprotti@una.ac.cr
}

Recibido 29-X-2001. Corregido 24-XI-2003. Aceptado 16-IX-2005.

\begin{abstract}
Species richness and diversity of a fish community in a temporal water body at Caño Negro National Wildlife Refuge, Costa Rica. We evaluated fish community, species richness and diversity in a temporal water body of Río Frío, Caño Negro National Wildlife Refuge. These evaluations were done for three categories of water levels: low (below $1.5 \mathrm{~m}$ ), intermediate (between 1.5 and $3.0 \mathrm{~m}$ ) and high (deeper than $3 \mathrm{~m}$ ). A total of 10264 individuals were collected (nine families, 18 genera and 21 species). The most abundant species were Poecilia gillii (37\%) and Astyanax aeneus (19\%) and the least abundant were Ophisternon aenigmaticum $(0.06 \%)$ and Rhamdia nicaraguensis $(0.05 \%)$. The highest values in diversity $\left(\mathrm{H}^{\prime}=2.07\right)$, in the inverse of the Simpson index $(1 / \mathrm{D}=6.2)$ and of the Berger-Parker $(1 / \mathrm{d}=4.2)$, were recorded in the deepest water category. Diversity differed clearly among water levels $(\mathrm{p}<0.001)$. The high and intermediate categories were the most similar (conglomerate analysis: $72.9 \%$ ). Out of the 21 species captured, only $O$. aenigmaticum constitutes a new record for the ichthyofauna of Río Frío in the Caño Negro National Wildlife Refuge. The temporal water body of the Río Frío, considered in this study in the Playuela sector of Caño Negro National Wildlife Refuge is a nonhomogeneous ichthyological system. Rev. Biol. Trop. 54(2): 639-645. Epub 2006 Jun 01.
\end{abstract}

Key words: fish, diversity, temporal water body, Río Frío, Caño Negro National Wildlife Refuge, Costa Rica.

El Refugio Nacional de Vida Silvestre Caño Negro (RNVSCN), ubicado en la región noroeste de Costa Rica $\left(10^{\circ} 54^{\prime} \mathrm{N}, 84^{\circ} 47^{\prime} \mathrm{W}\right)$ es una de las áreas protegidas de vital importancia para la protección de la flora y fauna de los humedales, así como para el mantenimiento de la calidad ambiental de la zona Caribe Norte, por su papel regulador de inundaciones y sostén de caudales de los principales ríos y caños (Pacheco et al. 1985).

El RNVSCN presenta un sistema de lagunas estacionales que varían en su profundidad y extensión según los cambios en el caudal del río Frío y sus tributarios; ésta variabilidad en el nivel del agua, afecta también la disponibilidad de hábitat para los organismos que allí se encuentran, en especial los peces, que constituyen un elemento importante en la trofodinámica del ecosistema (Mora et al. 1997, Cabrera Peña et al. 2003), y también como fuente de ingresos económicos y de alimentación para los habitantes de la zona (Cabrera et al. 1992, Méndez y Protti 2006).

Según Bussing (1998), en esta región se encuentran 49 especies de peces incluídas en 19 familias, de las cuales las más abundantes son Cichlidae, Poeciliidae y Characidae.

El objetivo de este trabajo fue determinar la diversidad, dominio y equidad de la ictiofauna de un cuerpo de agua temporal en el río Frío, sector Playuela, RNVSCN, Costa Rica, según el nivel de agua que le impone el ciclo hidrológico de este río. 


\section{MATERIAL Y MÉTODOS}

Este estudio se realizó entre setiembre de 1999 y setiembre de 2000, en un cuerpo de agua temporal en el río Frío, sector Playuela, RNVSCN (Fig. 1). El área corresponde a una llanura de inundación expuesta a fuertes variaciones en su profundidad y extensión, según las condiciones hidrológicas y a las estaciones del año (Boza 1988).

Se definieron tres categorías de profundidad: baja (menor a $1.5 \mathrm{~m}$ ), media (entre 1.5 y $3.0 \mathrm{~m}$ ) y alta (mayor de $3 \mathrm{~m}$ ), de acuerdo al registro de una torreta de medición de nivel de agua $( \pm 10 \mathrm{~cm})$, ubicada en la margen izquierda del río Frío para relacionar las capturas con las fluctuaciones en el nivel del agua en el sector Playuela.

La recolecta de peces se realizó por duplicado, utilizando un chinchorro de $25 \mathrm{~m}$ de largo y $1.5 \mathrm{~m}$ de ancho, con un copo central de $2 \mathrm{~m}$ de longitud y una luz de malla de $1 \mathrm{~cm}$. Los ejemplares capturados fueron fijados en una sol. de formaldehído $10 \%$ y su determinación taxonómica se realizó de acuerdo con Villa (1982) y Bussing (1998).

La diversidad de especies se determinó de acuerdo al índice de Shannon: $\mathrm{H}^{\prime}=-\sum \mathrm{p}_{\mathrm{i}} \ln \mathrm{p}_{\mathrm{i}}$, donde $\mathrm{p}_{\mathrm{i}}$ es la proporción de individuos de la "i" especie; y se procedió a establecer posibles diferencias significativas entre la diversidad de cada categoría de nivel de agua, para lo cual se determinó la varianza de cada H', así como el valor de " $t$ " y sus respectivos grados de libertad (Magurran 1988).

El índice dominancia de Berger-Parker, se derivó a partir de la ecuación: $\mathrm{d}=\mathrm{N}_{\max } / \mathrm{N}_{\mathrm{T}}$, donde: $\mathrm{N}_{\max }=$ el número total de individuos capturados de la especie más abundante y $\mathrm{N}_{\mathrm{T}}=$ número total de individuos capturados de todas las especies (Henderson y Seaby 1997). Con el propósito de establecer direccionalidad de este índice con H', los resultados son expresados según su inverso (Magurran 1988). Los cálculos de ambos índices se realizaron por medio del programa computacional Diversity vers. 1.2 (Henderson y Seaby 1997).

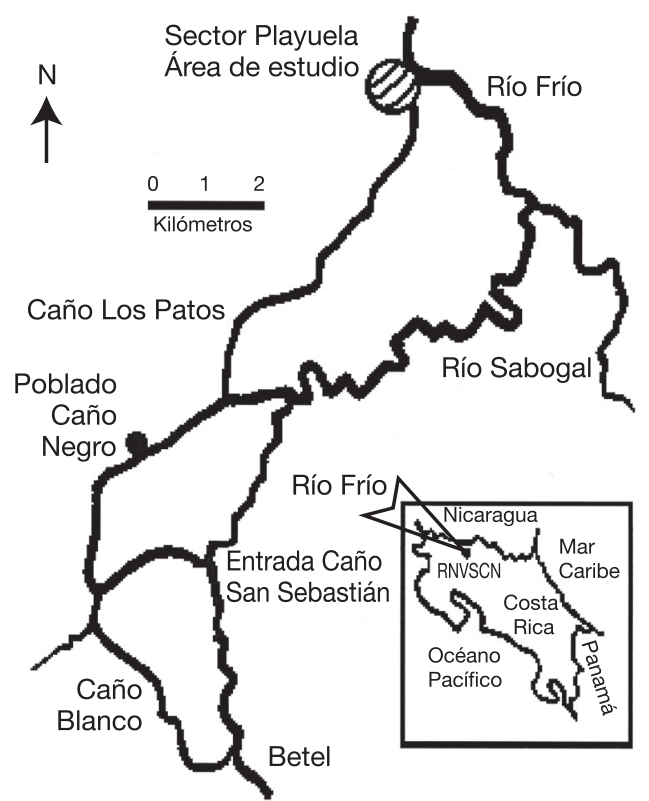

Fig. 1. Área de estudio: sector Playuela, RNVSCN.

Fig. 1. Study area in the Playuela sector of CNNWR.

La similitud de especies entre las categorías del nivel de agua, se efectuó según el análisis de agrupamiento de Morisita-Horn (Magurran 1988). El resultado de este análisis se graficó en un dendrograma de similitud, según el método de ligamiento simple (Pielou 1984), utilizando el programa computacional ACOM3. Bas, para calcular y resolver matrices de similitud (Navarro 1984).

\section{RESULTADOS}

De acuerdo con las categorías establecidas para el nivel de agua, el sector Playuela alcanzó niveles altos en siete meses no consecutivos; mientras que las categorías media y baja sólo se alcanzaron en tres meses para cada una de ellas. Los niveles extremos (máximos y mínimos) se registraron durante agosto y abril del 2000 , con $4.6 \mathrm{~m}$ y $0.4 \mathrm{~m}$ respectivamente (Fig. 2). 


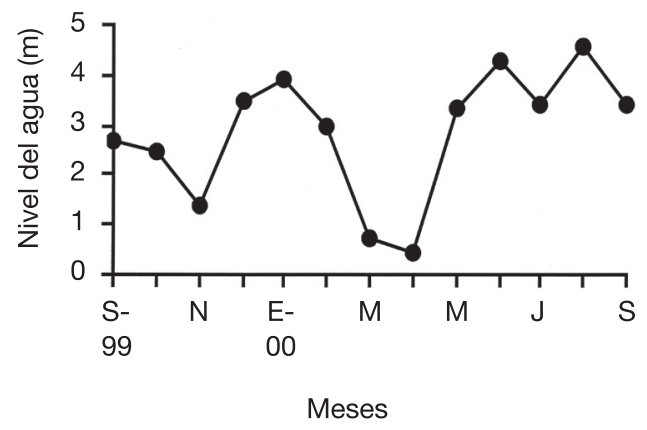

Fig. 2. Fluctuación mensual del nivel del agua en el río Frío, RNVSCN para el periodo 1999-2000.

Fig. 2. Monthly fluctuations of water level in the Río Frío, CNNWR for the period 1999-2000.

Se recolectó un total de 10264 individuos, pertenecientes a nueve familias, 18 géneros y 21 especies (Cuadro 1), siendo las familias Cichlidae y Characidae las que, presentaron el mayor número de especies, con nueve y cuatro respectivamente. Las especies con mayor abundancia numérica durante el período de estudio fueron: Poecilia gillii ( $\mathrm{n}=3$ 760), Astyanax aeneus ( $\mathrm{n}=1$ 900); mientras que los grupos menos abundantes correspondieron a Ophisternon aenigmaticum $(\mathrm{n}=6)$ y Rhamdia nicaraguensis $(\mathrm{n}=5)$.

En la categoría de nivel de agua alta se capturó el mayor número de especies $(\mathrm{n}=20)$. El $57 \%$ de la captura total se registró en la categoría baja; mientras que la menor abundancia se obtuvo en la categoría alta (19\%) (Fig. 3).

El mayor valor en la diversidad de especies se registró en la categoría alta $\left(\mathrm{H}^{\prime}=2.07\right)$, coincidiendo con los valores más altos en el inverso del índice de Berger-Parker $(1 / \mathrm{d}=1.7)$ (Cuadro 2). La diversidad de peces, en todas las categorías según el nivel de agua, mostraron diferencias estadísticas altamente significativas entre sí $(\mathrm{p}<0.001)$.

El análisis de agrupamiento reconoció a las categorías alta y media, como las más similares $(71.3 \%)$, en cuanto a la abundancia numérica de cada especie (Fig. 4).

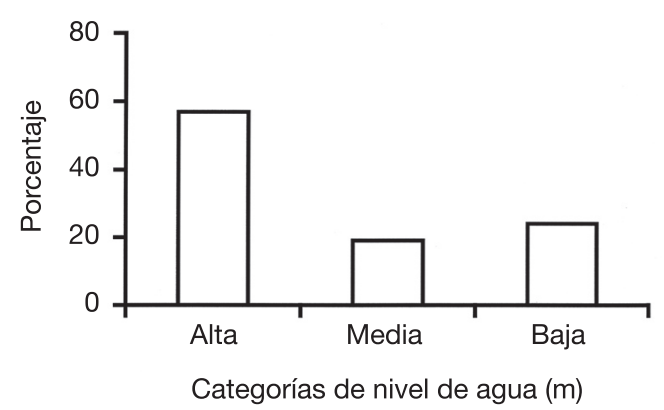

Fig. 3. Abundancia porcentual de las capturas por categoría de nivel de agua en el sistema temporal de agua en el río Frío, sector Playuela, RNVSCN.

Fig. 3. Abundance percentage of captures for the three water level categories in Playuela sector.

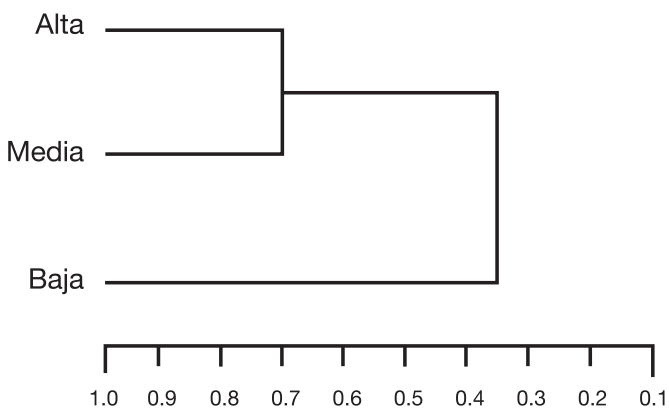

Fig. 4. Asociación de las categorías de nivel de agua según su composición ictiofaunística.

Fig. 4. Association for the three water level categories according to their ichthyofaunistic composition.

\section{DISCUSIÓN}

Las fluctuaciones en el nivel de agua del área de estudio, están directamente relacionadas con el régimen de lluvias y la dinámica de escorrentía en la cuenca del río Frío; sin embargo, la disminución del nivel no es simultánea con el descenso de las lluvias. El comportamiento atípico en el nivel de agua registrado en enero (3.9 m) en relación con la época seca (enero a abril) establecida por Brenes et al. (1996), puede ser atribuído a la influencia del 
CUADRO 1

Composición taxonómica y abundancia numérica de la ictiofauna en un cuerpo de agua temporal en el río Frío, sector Playuela, RNVSCN, según las categorías del nivel del agua

TABLE 1

Taxonomic composition and abundance of Ichthyofauna for three water level categories of a temporal water body of the Rio Frio, Playuela sector, CNNWR

Categorías de nivel

\begin{tabular}{|c|c|c|c|c|c|}
\hline Familia & Especie & Baja & Media & Alta & Total \\
\hline Lepisosteidae & Atractosteus tropicus & 54 & 16 & 24 & 94 \\
\hline Clupeidae & Dorosoma chavesi & 22 & 6 & 1 & 29 \\
\hline \multirow{4}{*}{ Characidae } & Astyanax aeneus & 175 & 1276 & 449 & 1900 \\
\hline & Carlana eigenmanni & 32 & 112 & 462 & 606 \\
\hline & Bramocharax bransfordii & 2 & 13 & 27 & 42 \\
\hline & Roeboides bouchellei & 242 & 392 & 317 & 951 \\
\hline \multirow[t]{2}{*}{ Pimelodidae } & Rhamdia guatemalensis & 8 & 20 & 2 & 30 \\
\hline & Rhamdia nicaraguensis. & & 5 & & 5 \\
\hline Poeciliidae & Poecilia gillii & 3394 & 120 & 246 & 3760 \\
\hline Atherinidae & Atherinella hubbsi & & 9 & 1 & 10 \\
\hline Symbranchidae & Ophisternon aenigmaticum & & & 6 & 6 \\
\hline \multirow[t]{9}{*}{ Cichlidae } & Amphilophus citrinellus & & 13 & 39 & 52 \\
\hline & Archocentrus centrarchus & 598 & 107 & 89 & 971 \\
\hline & Archocentrus nigrofasciatus & 132 & 38 & 8 & 178 \\
\hline & Astatheros longimanus & 505 & 78 & 59 & 642 \\
\hline & Astatheros rostratus & 521 & 161 & 26 & 708 \\
\hline & Herotilapia multiespinosa & 86 & 43 & 11 & 140 \\
\hline & Hypsophrys nicaraguensis & 11 & & 8 & 19 \\
\hline & Parachromis managuensis & 49 & 26 & 57 & 130 \\
\hline & Oreochromis niloticus & 3 & 8 & 25 & 36 \\
\hline Eleotridae & Gobiomorus dormitor & 1 & 3 & 5 & 9 \\
\hline
\end{tabular}

fenómeno atmosférico La Niña que prevalece desde 1998 (Anónimo 2001) y que posiblemente incidió en el aumento de la precipitación durante este mes en la parte alta de la cuenca del río Frío.

El número de familias y especies registradas en este estudio, fue menor a las informadas por Bussing (1998) para la cuenca del río Frío, pero superior a lo determinado por Campos (1986) (seis familias y 15 especies) y por
Castro (1991) (seis familias y 20 especies) en el RNVSCN.

La tolerancia a las variaciones ambientales y a la plasticidad genética que caracteriza a la familia Cichlidae (Pullin 1988, Urriola et al. 2004a, b, Peña-Mendoza et al. 2005), influye en que este taxón sea el mejor representado; ya que posiblemente, estos atributos le confieren una posición más ventajosa sobre los otros componentes de la ictiofauna en la zona en estudio. 
CUADRO 2

Valores de los indices de diversidad según las categorías de nivel de agua

TABLE 2

Values of diversity indexes for three water level categories

$\begin{array}{lccc}\text { Categoría } & \begin{array}{c}\text { Shannon } \\ \mathrm{H}^{\prime}\end{array} & \begin{array}{c}\text { Varianza } \\ \left(\mathrm{H}^{\prime}\right)\end{array} & \begin{array}{c}\text { Berger Parker } \\ 1 / \mathrm{d}\end{array} \\ \text { Baja } & 1.52 & 0.0003 & 1.7 \\ \text { Media } & 1.75 & 0.0008 & 1.9 \\ \text { Alta } & 2.07 & 0.0004 & 4.2\end{array}$

La dominancia numérica de $P$. gillii y $A$. aeneus, concuerda con los resultados de Castro (1991). La amplia distribución espacial que presentan estas dos especies, así como, su constante reproducción a través del año y la tendencia a formar cardúmenes (Bussing 1993, 1998), pueden explicar la alta densidad registrada.

El tamaño relativamente pequeño y su alta resistencia a los cambios físicos y químicos, podrían explicar el dominio de $P$. gillii (Chapman et al. 1991, Chapman et al. 1993). La condición dominante de ésta, también fue informada por Sánchez y Rueda (1999) para el complejo de Pajarales, colindante con la Ciénaga Grande de Santa Marta, Colombia.

La baja captura de $O$. aenigmaticum y $R$. nicaraguensis, puede ser atribuída a que la primera de ellas, es más activa durante la noche y tiende a permanecer enterrada en el día (Villa 1982); mientras que la segunda, a pesar de ser común en Nicaragua (Astorqui 1971, Villa 1982), no es muy común en la zona norte de Costa Rica (Bussing 1998). O. aenigmaticum constituye un nuevo registro para la ictiofauna de Caño Negro; sin embargo, Rosen y Greenwood (1976 fide Bussing 1998), sugirieron su presencia en Costa Rica.

La mayor cantidad de especies capturadas en la categoría alta, sugiere una fluctuación temporal de la ictiofauna en la zona de estudio, lo que coincide con lo informado por Oldani y Tablado (1985) en la laguna La Cuarentena, río Paraná. Esta relación resalta la importancia del área disponible en la historia natural de los peces, ya que disponen de una superficie mayor para su alimentación, reproducción y otros aspectos fisiológicos, como sucede, con el gaspar cuyo índice gonadosomático y desplazamientos reproductivos en Caño Negro, son mayores durante los meses más lluviosos (Campos 1986, Mora et al. 1997, Bussing 1998).

El valor más alto en la diversidad de peces en el sector Playuela, en la categoría alta, puede ser explicado por la mayor disponibilidad de espacio que facilita un mejor desplazamiento desde el cauce principal del río a las áreas de inundación temporal, y por lo tanto, una mejor distribución de ellas. Esta alta diversidad también está relacionada con la baja dominancia, expresada según el inverso del índice de dominancia de Berger-Parker, donde las dos especies mejor representadas (A. aeneus y Carlana eigenmanni), aportaron el $46 \%$ de la captura total; mientras que, en las categorías media ( $A$. aeneus y Astatheros rostratus) y baja ( $P$. gillii y Archocentrus centrarchus), representaron el $59 \%$ y $68 \%$ respectivamente.

Los valores de H' obtenidos en este estudio coinciden con los estimados por Margaleff (1974) para varios taxones de peces y fueron mayores a los registrados por Castro (1991) en otros sectores del RNVSCN, y por Sánchez y Rueda (1999) en su análisis espacio-temporal de las poblaciones de peces en un estuario del Caribe colombiano.

El valor más bajo en la varianza de H' para el sector Playuela, correspondió con la menor diversidad registrada durante todo el periodo de estudio, contrastando con la tendencia al aumento de varianza de H' en sitios con menor diversidad informada por Álvarez et al. (1990).

Las diferencias observadas según el nivel de agua sugieren una alta influencia de las condiciones ambientales sobre la comunidad de peces, lo que podría condicionar la permanencia de las especies menos tolerantes e incidir en la variación de la diversidad y dominancia de especies (Mahon y Balon 1977), lo cual podría explicar la dominancia de $P$. gillii, A. centrarchus, Astatheros longimanus y A. rostratus durante los periodos más secos (Chapman et al. 1991, Chapman et al. 1993 y Bussing 1998). 
De acuerdo con el análisis de agrupamiento, la categoría baja fue la que mostró mayor diferencia con las otras, debido a la gran abundancia de P. gillii, A. centrarchus, $A$. longimanus y $A$. rostratus. La similitud en las categorías alta y media, puede ser atribuída a las 18 especies que comparten y a la abundancia numérica, principalmente de $C$. eigenmanni, Roeboides bouchellei, P. gillii y A. centrarchus que influyeron en el grado de semejanza detectado.

De acuerdo con los índices de diversidad utilizados, se puede concluir que el cuerpo de agua temporal en el sector Playuela, RNVSCN, corresponde a un sistema cuya ictiofauna fluctúa temporalmente.

\section{RESUMEN}

Se evaluó la diversidad, el dominio y la equidad de la ictiofauna en un cuerpo de agua temporal en el río Frío, sector Playuela, Refugio Nacional de Vida Silvestre Caño Negro, según tres categorías del nivel de agua (baja [menor a $1.5 \mathrm{~m}$ ], media [entre 1.5 y $3.0 \mathrm{~m}$ ] y alta [mayor de 3.0 $\mathrm{m}]$ ). Se recolectaron 10264 individuos, pertenecientes a nueve familias, 18 géneros y 21 especies. Las especies que aportaron el mayor número de individuos fueron Poecilia gillii (37\%) y Astyanax aeneus (19\%); mientras que las menos abundantes fueron Ophisternon aenigmaticum $(0.06 \%)$ y Rhamdia nicaraguensis $(0.05 \%)$. El valor más alto en la diversidad de especies se registró en la categoría alta $\left(H^{\prime}=2.07\right)$; coincidiendo con los valores más altos en el inverso del índice de Simpson $(1 / \mathrm{D}=6.2)$ y de BergerParker $(1 / \mathrm{d}=4.2)$. De acuerdo con su diversidad, todas las categorías consideradas para el nivel de agua, mostraron diferencias estadísticas altamente significativas entre sí $(\mathrm{p}<0.001)$. El análisis de conglomerados reconoció a las categorías alta y media como las más similares (72.9\%). De las 21 especies capturadas, solamente $O$. aenigmaticum constituye un nuevo registro para la ictiofauna de Caño Negro. Este cuerpo de agua estacional es un sistema cuya ictiofauna fluctúa temporalmente.

Palabras clave: peces, comunidad, composición de especies, diversidad, dominancia, lagunas estacionales, río Frío, Refugio Nacional de Vida Silvestre Caño Negro, Costa Rica.

\section{REFERENCIAS}

Álvarez, B., F, Linares \& M. Alvarez. 1990. Análisis de la diversidad, amplitud y traslape del nicho en la comunidad de peces del sistema Teacapan - Agua Brava, Nayarit, México. An. Inst. Cienc. Mar Limnol. Univ. Nal. Autón. México. 17: 215-240 p.

Astorqui, I. 1971. Peces de la cuenca de los grandes lagos de Nicaragua. Rev. Biol. Trop. 19: 7-57.

Boza, A. 1988. Costa Rica National Parks. Heliconia, Neotrópica. San José, Costa Rica. 70 p.

Brenes, L., F. Solano, D. Salas \& V. Cortés. 1996. Procesos hidrodinámicos de sedimentación en el sistema lagunar Caño Negro. Informe I. Programa de investigación geográfica, Escuela de Geografía, Universidad de Costa Rica, San José, Costa Rica. 126 p.

Bussing, W.A. 1993. Fish communities and environmental characteristics of tropical rain forest river in Costa Rica. Rev. Biol. Trop. 41: 791-809.

Bussing, W.A. 1998. Peces de las aguas continentales de Costa Rica. Universidad de Costa Rica, San José, Costa Rica. 478 p.

Cabrera, J., C. Ampié \& G. Galeano. 1992. Presencia de Oreochromis niloticus (Pisces: Cichlidae) en las lagunas estacionales del Refugio Nacional Caño Negro, Costa Rica. Brenesia 38: 169-170.

Cabrera Peña, J., M. Protti Quesada, M. Urriola Hernández \& R. Cubero Murillo. 2003. Distribución y abundancia de Caiman crocodilus en el Refugio Nacional de Vida Silvestre Caño Negro, Costa Rica. Rev. Biol. Trop. 51: 571-578.

Campos, J. 1986. Estudio sobre los recursos pesqueros de las lagunas de inundación de Caño Negro. Biocenosis 3: 24-26.

Castro, M. 1991. Composición, diversidad y abundancia de la fauna íctica en la laguna del Refugio de Vida Silvestre Caño Negro. Investigación por tutoría para optar al grado de Bachiller en Biología Marina, Universidad. Nacional, Heredia, Costa Rica. 24 p.

Chapman , L., D. Kramer \& C. Chapman. 1991. Population dynamics of the fish Poecilia gillii (Poecilidae) in pools of an intermittent tropical stream. J. Anim. Ecol. 60: 441-453. 
Chapman, L. \& C. Chapman. 1993. Desiccation, flooding, and the behavior of Poecilia gillii (Pisces: Poeciliidae). Ichthyol. Explor. Freshwater 4: 279-287.

Henderson, P.A. \& R.M.H. Seaby. 1997. Species diversity and richness. Projeto Mamiraúa. Lymington, Inglaterra. Ver. 1.2.

Magurran, E.A. 1998. Ecological diversity and its measurement. Princeton University, Princeton, Nueva Jersey, EEUU. 169 p.

Mahon, R. \& E. Balon. 1977. Fisheries research community structure in lakes shore lagoons on long point, Lake Erie, Canada. Environ. Biol. Fish. Res. 18: 71-82.

Margalef, R. 1974. Ecología. Omega, Barcelona, España. $951 \mathrm{p}$.

Méndez E., C. \& M. Protti Q. 2006. Actividad pesquera artesanal en un sistema de lagunas estacionales en la zona norte de Costa Rica. Rev. Biol. Trop. 54: in press.

Mora, M., J. Cabrera \& G. Galeano. 1997. Reproducción y alimentación del gaspar Atractosteus tropicus (Pisces: Lepisosteidae) en el Refugio Nacional de Vida Silvestre Caño Negro, Costa Rica. Rev. Biol. Trop. 45: 861-866.

Navarro, R. 1984. Programa computacional para el análisis numérico de comunidades. Medio Ambiente, Chile 7: 82-87.

Oldani, N.O. \& A. Tablado. 1985. Dinámica temporal de pequeños peces de agua libre en la Laguna "La Cuarentena" (Isla Carabajal, Río Paraná medio). Stud. Neotrop. Fauna Environ. 20: 49-58.

Pacheco, A., M. Rodríguez, E. López \& G. Canessa. 1985 Plan operativo. Refugio Nacional de fauna Silvestre Caño Negro. Subdirección General de Vida Silvestre, Ministerio de Agricultura y Ganadería. San José, Costa Rica. 130 p.
Peña-Mendoza, B., J.L. Gómez-Marquéz, I.H. SalgadoUgarte \& D. Ramírez-Noguera. 2005. Reproductive biology of Oreochromis niloticus (Perciformes: Cichlidae) at Emiliano Zapata dam, Morelos, Mexico. Rev. Biol. Trop. 53: 515-522.

Pielow, E.C. 1984. The interpretation of ecological data. A primer on classification and ordination. Wiley, Nueva York, EEUU. 263 p.

Pullin, R. 1988. Tilapia genetic resources for aquaculture. International Center of Living Aquatic Resources Management, Manila. 108 p.

Sánchez, C. \& M. Rueda. 1999. Variación de la diversidad y abundancia de especies ícticas dominantes en Delta del Río Magdalena, Colombia. Rev. Biol. Trop. 47: 1067-1079.

Urriola Hernández, M., J. Cabrera Peña \& M. Protti Quesada. 2004a. Composición, crecimiento e índice de condición de una población de Poecilia reticulata (Pisces: Poeciliidae), en un estanque en Heredia, Costa Rica. Rev. Biol. Trop. 52: 157-162.

Urriola Hernández, M., J. Cabrera Peña \& M. Protti Quesada. 2004b. Fecundidad, fertilidad e índice gonadosomático de Poecilia reticulata (Pisces: Poeciliidae) en un estanque en Santo Domingo, Heredia, Costa Rica. Rev. Biol. Trop. 52: $945-$ 950 .

Villa, J. 1982. Peces nicaragüenses de agua dulce. Colección Cult. Banco de América, Ser. Geogr. Natur. 3: 253 p.

\section{REFERENCIA DE INTERNET}

Anónimo. 2001. Aspectos sobre el fenómeno de la "Niña". 6 p. (consultado 7 enero 2006, http://www.imn.ac.cr). 
